\title{
A Depopulation Embedded System Model in Urban Environments
}

\author{
C. Wright Brown \\ School of Science Information Department of Electrical and \\ Arts and Technologies \\ University of Baltimore \\ Baltimore, USA \\ cwrightbrown@ubalt.edu \\ K. Nyarko \\ Computer Engineering \\ Morgan State University \\ Baltimore, USA \\ kofi.nyarko@morgran.edu
}

\author{
D. Karimou \\ Science \& Technology Department \\ \& LICEF Research Center \\ TELUQ, University of Quebec \\ Montreal, Canada \\ do_zak@hotmail.com
}

\author{
H. Saliah-Hassane \\ Science \& Technology Department \\ \& LICEF Research Center \\ TELUQ, University of Quebec \\ Montreal, Canada \\ saliah-hassane.hamadou@teluq.ca
}

\begin{abstract}
The threat of terrorism is a constant reminder of the significance of developing effective strategies to safeguard the population. This is particularly important in large, densely populated areas, which can often be the primary targets of a terrorist attack. In such a situation, the implementation of a depopulation plan becomes very challenging to manage. This paper explores one possible method of addressing rapid evacuation of densely populated urban environments in response to a terrorist threat through an in-vehicle embedded system model. The objective of the model is to determine feasible technologies that enable mobile ad-hoc mesh communication to safely depopulate an urban area.
\end{abstract}

Key words: embedded system, data acquisition, GPS, cooperative system, depopulation, homeland security

\section{INTRODUCTION}

With the September 11th, 2001 terrorist attacks came many changes to national security efforts. Many security measures have been implemented at all major points of entry into the United States. These efforts include: vulnerability and risk assessments, stiff access control, reinforced security presence, routine emergency drills, detailed security plans, and scheduled training exercises. This research considers an embedded system model, which integrates existing sensors and technologies to create ad-hoc wireless communications networks between vehicles. This study will present scenarios in which real-time traffic conditions are gathered and communicated to vehicles during a rapid depopulation procedure through an ad-hoc mesh network. With realtime analysis and communication of traffic conditions, vehicles can be properly routed around congestions points in order to streamline the evacuation process. Traffic information such as vehicle speed and position will be relayed over the mobile ad hoc network and eventually through access points connected to processing and analysis servers, which will generate appropriate routing information for each vehicle, which will then be sent back across the network. In this environment each vehicle communicates with the server, in near real-time, over multiple ports. The data is collected by a central server, with redundant servers, where the information is processed and returned to each node. In this scenario, each vehicle acts as a data processing and communications node in the network and can perform one of the following actions: (1) display pertinent routing information to driver, (2) gather vehicle state information, (3) relay information to/from other nodes or the access point.

As rapid changes in technology increase, companies will need to invest and innovate to accommodate the demands in international markets, globalization, access collective knowledge, and enhance customer expectations. An important part of these investments are advances in transportation, which links a driver to their vehicles and traffic environments. This depopulation embedded system model could be expanded into a number of smart transportation applications in the event of a homeland security urban evacuation. The critical role that these technologies can provide for a driver would include: collision detection, pedestrian detection, management of the environment, interactions with the driver for rapid decision making to cope with contingencies. Similarly, the technologies offered by mobile devices such as mobile networks, GPS technologies, communication protocols between vehicles, broadcast and communicate information about road traffic would be feasible. It is also possible to exchange information with a driver to increase their perception and behavior; drivers can also collaborate to regulate the traffic. To make use of these technologies several scenarios can be considered, such as collision detection, vehicle positioning, increasing environment awareness, lane departure detection, and detection of driver drowsiness. This model will suggest various vehicle embedded technologies that can be used for managing the collection of traffic information, communications and interactions between drivers for the purpose of coordinating rapid vehicle depopulation.

\section{ANTI-TERRORISM AND SECURITY}

Terrorists are opportunistic and flexible. They learn from experience and modify their tactics and targets to exploit 
perceived vulnerabilities and avoid observed strengths. As security increases around more predictable targets, terrorists shift their focus to less protected assets. Therefore, enhancing countermeasures for any one terrorist tactic or target makes it more likely that terrorists will favor another. However, terrorists tend to target critical infrastructures to achieve three general types of effects that make transportation nodes more likely than other possible targets.

1. Direct infrastructure effects: cascading disruption or arrest of the functions of critical infrastructures or key assets through direct attacks on a critical node, system, or function.

2. Indirect infrastructure effects: cascading disruption and financial consequences for government, society, and economy through public and private sector reactions to an attack.

3. Exploitation of infrastructure: exploitation of elements of a particular infrastructure to disrupt or destroy another target.

This perspective would suggest that the mass transit system should not be relied upon as the primary method of achieving rapid depopulation. Furthermore, mass transit is regulated by various agencies, and these agencies must communicate and work together effectively to allow transit security to work as a system rather than in separate modes. However, mass transit is funded and managed at the local level and operated as a not-for-profit entity, which adds a further layer of administrative complexity to security issues. Moreover, mass transit systems were designed for openness and ease of public access, which makes monitoring points of entry and exit difficult, which in turn makes them more appealing targets.

In light of the vulnerabilities of mass transit systems, it would appear that depopulation through personal vehicles would be preferable. There are often several routes out of a major city, which is essential for ensuring continued evacuations in cases where damage has occurred to certain transit infrastructure points. However, several problems currently exist for achieving effective rapid depopulation with personal vehicles on public roads. In an emergency situation, the most common routes out of the city quickly become congested, preventing further evacuation efforts. Due to stress factors, there is often an increase is accidents during this time, which further exacerbates traffic conditions. A system that can mitigate these effects by properly routing vehicles based on real-time traffic feedback as well as minimize traffic incidents would enable current roadway infrastructures to serve as viable means for rapid depopulation.

\section{BACKGROUND}

Over the last decade, the technologies that enable integrated circuits and devices to connect, send, and store information have become prevalent. For example, the use of mobile devices has exploded within the last decade. It is quite reasonable to assume that a majority of passengers would possess a mobile device of some type. In addition, embedded systems have become cheaper to manufacture and more ubiquitous in modern day vehicles. As such, there has been an increase in research efforts to leverage these mobile and embedded computing devices to improve various aspects of driver/vehicle interaction as well as to aid drivers better interact with their external environment. In France, project ARCOS, which is a research project for secure driving [1], aimed to manage the distances between vehicles, prevent crashes, and monitor vehicles for erratic behavior was largely based on embedded computing. Similarly, the U.S. Department of Transportation launched a project called Intelligent Vehicle Initiative (IVI) to improve transportation safety and productivity by accelerating the phased deployment of intelligent transport [2]. In Japan, the launch of the Smart Cruise Systems, which resembles a similar U.S. program, has two distinct functions: Advanced CruiseAssist Highway Systems and Advanced Safety Vehicle $[3,4]$. All these efforts to improve transit safety are based on the availability of robust and inexpensive embedded or mobile computing devices. This research seeks to apply similar advances in computing to emergency evacuation.

\section{MODEL RESEARCH}

The proposed model consists of embedded devices in vehicles for traffic management, communications, and even vehicle control. This model relies on an embedded systems architecture combined with mesh networking technologies. The model is based on protocols that allow vehicles to communicate and interact in traffic, where the protocols are used to monitor and manage the distance between vehicles to build an internal model of the current state of traffic as well as prevent accidents, which would further exacerbate traffic conditions during an evacuation. Through various technologies and mobile networks, vehicles are able to communicate with one another as well as with a central server. 
This model also considers the use of mobile devices to collect, processes, and analyze data to support decisionmaking. The model also considers the use of available vehicle proximity sensors to better understand traffic conditions and decrease the potential for traffic incidents. Through the use of inexpensive embedded control devices, vehicles can be easily equipped with proximity sensing capabilities that enable emergency breaking.

\section{MODEL ENVIRONMENT}

For this work, vehicles that are equipped with various sensors and GPS devices were considered. In addition, a communication infrastructure that consists of the following three features were considered:

- A mobile ad hoc network that allows the vehicle to communicate and cooperate

- An on-board device that interfaces with key vehicle systems

- A device and program that allows transmission of data to a processing server

Mobile technologies such as WiFi networks and ad hoc mobile networks offer substantial opportunities for nodes operating in a single environment to communicate and cooperate to regulate traffic. For this work, nodes refer to a vehicle equipped with networking capabilities. This model also makes use of Mobile Ad-hoc Networks (MANET). Unlike wired networks, a mobile ad hoc network represents a distributed system formed by entities, which are connected by mobile wireless technologies. This network is a temporary system, without any existing infrastructure or centralized administration. Network nodes are deployed in environments without using any fixed support. Nodes must be able to self-organize and cooperate to provide services. This is the network approach used for this research as indicated in Figure 1.

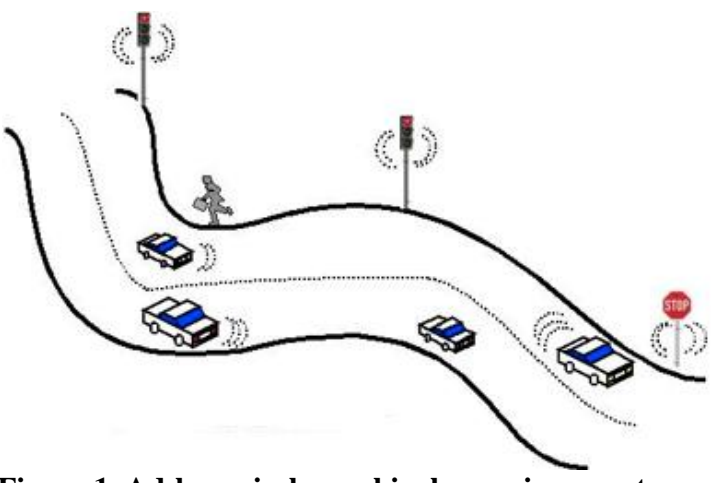

Figure 1. Ad-hoc wireless vehicular environment
The transmitter node is often required to go through other nodes to relay transmissions. In this case, each node in the system may act as a processing or relay node. In order to accomplish this, we must first find a method to solve the problem posed by the partitioning of nodes into several clusters with elements having the same common properties. One solution is to partition nodes into clusters, where for each cluster, a cluster head is chosen and responsible for managing communication or sometimes to broadcast information or messages outside its area of collaboration. Cluster members are determined by the physical proximity of vehicular nodes as well as the density of nodes in a cluster. An additional property that affects cluster members is communication bandwidth.

In part, this research explores clustering techniques to group similar nodes into several groups or clusters. Several clustering algorithms have been proposed in the past, with multiple approaches with varying results. In this work, we propose an efficient method for grouping nodes to obtain clusters formed by nodes with the same area properties. The main idea of this grouping allows nodes with similar properties to communicate and cooperate with each other using the channel of a cluster. Subsequently, to ensure service requests in the other clusters, protocols and bridges can be established to allow communications between clusters as seen in Figure 2.

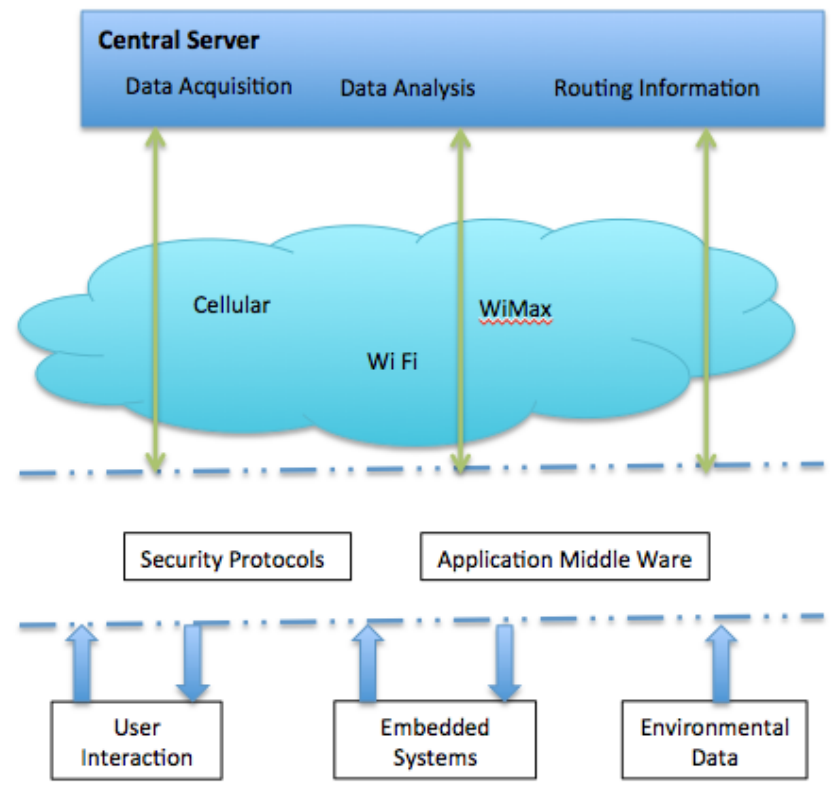

Figure 2. System architecture 
The CompactRIO serves as the on-board device that directly interacts with key systems onboard the vehicle. This device can be combined with several other possible embedded sensors on the vehicle to detect and trace various events. Lastly a proximity sensor and embedded system can be used to automate emergency breaking as well as report information to a processing server. A depiction of the overall mobile architecture is displayed in Figure 3.

\section{APPROACH}

The CompactRio [5] is a system control and data acquisition device. It is a real-time embedded processor for autonomous operations and reliable distributed communication. It is possible to adapt this device to acquire information from electronic and mechanical systems on the vehicle. Given these advantages, the CompactRio can be embedded in a vehicle for monitoring, data acquisition, and interfacing with mobile communication devices. In addition the CompactRio can be interfaced with LabView, which is ideal for development and testing. Once this module is installed in a vehicle, it is possible to remotely control key electrical and mechanical systems within the vehicle. Therefore, by using the LabView software and a CompactRIO device, a system can be implemented using wireless sensors that collect information on the vehicle and communicates with a central server. Sending data to the server is done in different ways. One method is to setup an ad hoc network that relays information, node by node, to the server. Another is to rely on existing infrastructures such as $\mathrm{WiFi}$ or WiMax networks. Another method is to use $\mathrm{Wi}-\mathrm{Fi}$ access points that serve as relays for data transmission and communications between sensors, such as Wi Fi or WiMax and the processing server. The objective is the transmission of data, which can be analyzed and processed. Based on outcomes, it is possible to interact with the vehicle parameters by injecting information into the system. In effect information from the entire vehicle network can initiate action within one or more vehicles as necessary.

When a communication infrastructure is not available, nodes are partitioned automatically into groups, which communicate in an ad-hoc fashion. The approach to partitioning the mobile environment is structured as follows:

- Clusterhead-formation is executed among the nodes that self-proclaimed as server nodes. These nodes are able to connect to central server using existing infrastructure or cellular connections. This method is based on a modified clustering method outlined by Basagni [6] where the environment of this project is taken into account.

- Cluster-choice operated by each participant to join a cluster. The main purpose of this step is to partition the network into clusters. This process produces a system composed by a set of clusters with arbitrary elements. The goal of clustering is to partition entire network into different parts. Each part is formed by a set of connected nodes (clusterhead, ordinary nodes) that provide broadcasting information on the network or on a cluster without all the nodes participate in this operation.

Consider the graph $\mathbf{G}(\mathbf{V}, \mathbf{E})$, which represents indirect communications network, where $\mathbf{V}$ is the set of mobile nodes $\mathbf{V}=\{\mathbf{v} \mathbf{1}, \mathbf{v} \mathbf{2}, \ldots \mathbf{v p}\}\{|\mathbf{V}|\}$ and $\mathbf{E}$ is the set of edges. We consider that each node vi has a positive weight (wi). For two nodes vi, vj, wi \# wj. Similarly, we consider for each pair of nodes vi, vj, edge eij = eji. In other words, the connections between two edges are bidirectional (symmetric graph). Clusters are notes $\mathbf{C i}$ for $\mathbf{1} \leq \mathbf{i} \leq \mathbf{k}$ and the clusterhead $\mathbf{C H i}$ for $\mathbf{1} \leq \mathbf{i} \leq \mathbf{k}$. The clustering process divides $\mathbf{V}$ into a set of $\mathbf{k}$ subsets $\{\mathbf{V 1}$, $\mathbf{V} \mathbf{2}, \ldots \mathbf{V k}\}$ where $\mathbf{V}=\mathbf{U k i}=\mathbf{1} \mathbf{V i}$, so that each subset $\mathbf{V i}$ form a connected graph $\mathbf{G}$. And each subset represents a cluster.

Each node knows its degree, its weight, and the degrees of the nodes in its neighborhood. At the end of clustering process, each clusterhead knows the degree of all other clusterheads. The clustering process is carried out in two steps:

Step 1: Choosing the clusterhead. This first phase selects a clusterhead for each partition. Each cluster is composed of a clusterhead node leader and a set of ordinary nodes. The choice of clusterhead is based on the degree associated with each node. In case of equality, we refer to the weights of nodes to decide between. In this particular case, the node with the highest weight becomes a clusterhead. In this way, nodes with larger degrees initialize the clustering process by broadcasting a message to their neighbor who announces their wish to be clusterhead.

If a node $\mathbf{u}$ receives a message from a node $\mathbf{v}$ and the degree of $\mathbf{v}$ is greater than $\mathbf{u}$, then $\mathbf{u}$ is resigned and considers $\mathbf{v}$ a clusterhead. One node I becomes a clusterhead if at least one of the following conditions is met:

- $\mathbf{i}$ is the largest degree among its 1-neighborhood (close to a jump). Or 
- $\mathbf{i}$ is not the node with the highest degree in its 1neighborhood, but all its neighbors belong to 1 -jump to other clusters

Step 2: choice of ordinary nodes. For a node $\mathbf{v}$ with degree less than all its 1-neighborhood, two situations arise:

- If $\mathbf{u}$ is a node in its 1-neighborhood as clusterhead, then the node $\mathbf{v}$ is located in the cluster formed by $\mathbf{u}$.

- If his neighbor $\mathbf{u}$ greater degree belongs to another cluster, then two cases are possible. If it exists in its 1neighborhood, another clusterhead then $\mathbf{v}$ moves in this cluster. If not, then $\mathbf{v}$ creates its own cluster.

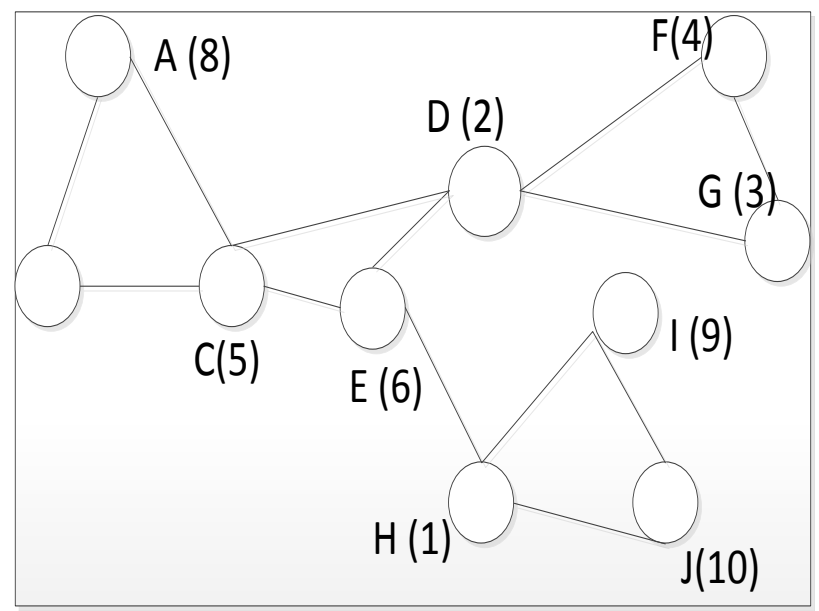

Figure 3. Initial network environment with vehicular nodes

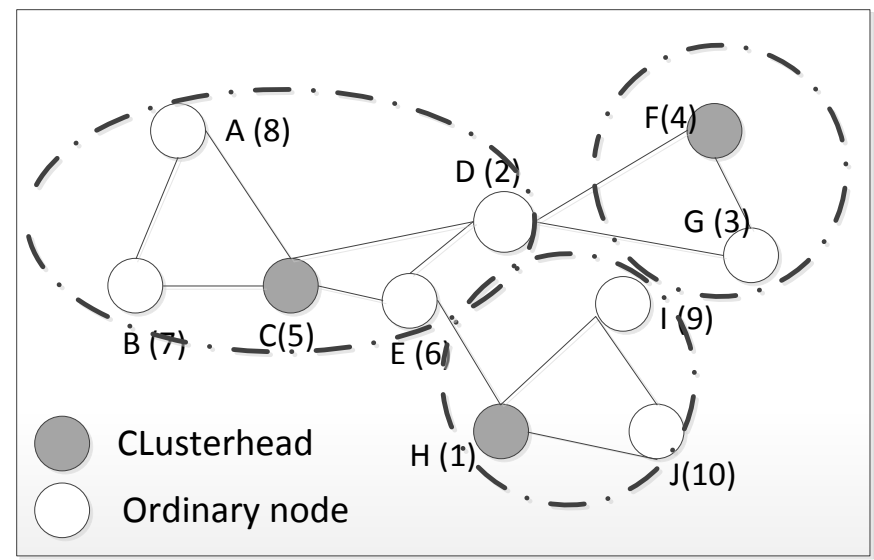

Figure 4. Automatic cluster partitioning

For example, the algorithm (see Figure 3 and 4) creates two non-overlapping clusters in which each node selects a clusterhead to joint among its neighbors. At the end of the process, the following conditions are satisfied:
- The entire network is partitioned into clusters. Each node belongs to exactly one cluster. A node is either a clusterhead or an ordinary node directly connected to a clusterhead with the higher level in its 1-neighborhood;

- Each cluster has a degree equal to $\mathbf{2}$ and each node is located at a clusterhead or breaks its clusterhead;

- At the end of the process, each clusterhead knows the number of nodes that are part of the cluster. Also, device nodes which connect the cluster to its neighboring nodes called edges are known by the two clusters they belong. Indeed, edge nodes are the stations that make the junction between two clusters and probably communicate with the other edge nodes in other clusters. These nodes provide translation of internal and external information to be sent or received clusters.

- Each node usually has at least one clusterhead in its 1neighborhood and belongs to the cluster formed by its neighboring node with the highest degree;

- Two clusterheads can never be ordinary neighbors and each node is one hop from its clusterhead.

\section{SIMULATION RESULTS}

Preliminary simulations were performed to determine if the approach outlined in this paper would reduce congestion and increase depopulation rates of an average city with multiple available routes. In the simulation, vehicles with direct internet access (over WiFi, WiMax or cellular) underwent standard protocol initialization as in [7]. Following this initialization all vehicles located in a given area are identified as shown in the communication archicture in Figure 5. The methodology uses socket technologies to simulate communications between vehicles and the server to update information in the database. Communications between the central server and vehicles are simulated as a response issued by the server to the vehicle, which makes query request to the database to update the vehicles position and status. This also makes it possible for the server to send routing information to vehicles running in an area.

The simulation begins with random distribution of vehicles on several routes, where the density of traffic is dependent on the number of lanes available for a given route. The vehicular network is partitioned into clusters ensuring all vehicles can effectively communicate with 
the central server. When an emergency state is set, the system sends a customized evacuation route to each vehicle based on their current position reported by onboard GPS. The simulation utilizes simulated proximity sensor data to ensure proper separation between vehicles to minimize traffic incidents. In addition, this sensor data is fed back to the central server to provide an indication of the stress levels of drivers (aggressive driving). This data can also be obtained from the breaking and acceleration patterns of the drivers. These stress levels are taken into account when determining appropriate routing data. In addition the system regulates when traffic should be directed in both directions on highway systems to maximize vehicle throughput. Preliminary results indicated a significant increase in depopulation rates over unassisted tradition vehicular evacuations. A future paper will detail the simulation setup and quantify the improvement in depopulation rates.

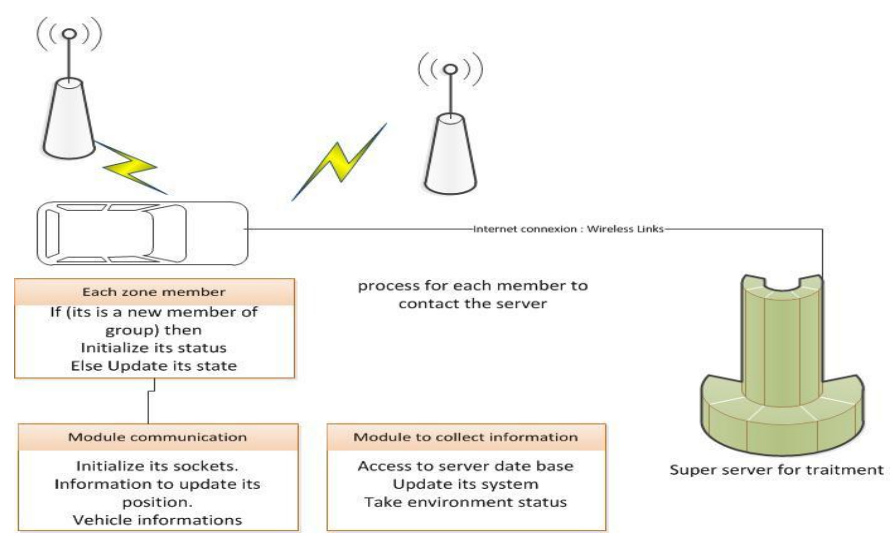

Figure 5. Vehicle communication architecture

\section{SUMMARY}

This model presents solutions to improve rapid depopulation, security and reliability of vehicles during a large scale terrorism event. The research offers technologies to automate and optimize the scenarios presented. These types of technologies will contribute to transportation research for smart vehicles using embedded systems.

\section{REFERENCES}

[1] INRIA. Les transports intelligents et l'aide à la conduite. Dossier de presse "Les véhicules intelligents" réalisé à l'occasion du symposium IV'2002, 18 au 20 juin 2002, Versailles, France.
[2] KLINGENBERG B. Opportunities for progressive deployment of intelligent transportation systems/ automated highway system technologies in support of commercial vehicle operations. Annual Meeting of the Transportation Research Board No77, ETATS-UNIS (01/1998). 1998, no 1651 (129 p.) (2 ref.), pp. 107-110

[3] D. Faudry les transports intelligents, Synthèse innovations transports du 25/11/04

[4] High-Tech for "Flying on the Ground, ThyssenKrupp AG; Transrapid International - A joint company of Siemens and ThyssenKrupp, August 16, 2002

[5] National instrument. Home Page (2009). Lien : http://www.ni.com/compactrio/whatis.htm

[6] S. Basagni. Distributed clustering for multihop wireless networks. In A. Annamalai and C. Tellambura, Proceedings of the IEEE International Symposium on Wireless Communications (ISWC'99), pp. 41-42, Victoria, BC, Canada, June 3-4 1999.

[7] Karimou, D., Myoupo, J. F. (2005), « An Application of an initialization Protocol to Permutation Routing in a Single-hop Mobile Ad-hoc Networks », The Journal of Supercomputing, March, vol. 31, no. 3, pp.

215-226(12) 